\title{
Understanding CO2 Mineralization in Interfacial Water Nanofilms
}

\author{
SIAVASH ZARE AND MOHAMMAD JAVAD \\ ABDOLHOSSEINI QOMI ${ }^{1}$
}

${ }^{1}$ Depertment of Civil and Environmental Engineering, University of California, Irvine, USA. 92617.

Carbonation of natural earth-abundant and synthetic metal silicates promises scalable solutions to permanently store $\mathrm{CO}_{2}$. With enigmatic observations undermining the ratelimiting role of dissolution in carbonation, understanding the kinetics proves critical in designing secure and economical geological carbon sequestration and concrete technologies. Here, we use atomistic simulations and density functional theory to probe the nature of physicochemical processes at the rock-water- $\mathrm{CO}_{2}$ interface. We show that while nanometerthick interfacial water films persist at unsaturated conditions consistent with in situ infrared spectroscopy, hydroxylated metal silicate surfaces catalyze $\mathrm{CO}_{2}$ speciation. Subsequently, through a proton hole transport between the bicarbonate and surface hydroxides, a carbonate is produced and directly precipitates on the surface to form a metal carbonate core. This leads to a ligand-assisted dissolution mechanism whose corresponding activation energy barrier is comparable with in operando X-ray diffraction measurements. These findings suggest hydrated metal silicate surfaces are more basic than appreciated and long-range interfacial proton hole mechanisms govern the early-stage carbonate nucleation in low-water systems. 